\title{
Internet Public Opinion Management in Big Data Era
}

\author{
Yongchang Ren ${ }^{1, a}$, Zhe $\mathrm{Li}^{2, \mathrm{~b}}$ \\ ${ }^{1}$ College of Information Science and Technology, Bohai University, Jinzhou, 121013, China \\ ${ }^{2}$ College of International Exchange, Bohai University, Jinzhou, 121013, China \\ arycryc@sina.com, b503412508@qq.com
}

Keywords: big data era; Internet public opinion management; new features; new challenges; path selection

\begin{abstract}
The profound changes in the network public opinion in the era of big data, based on the thinking and technology of big data, and the innovation of online public opinion management, have become an important part of promoting the modernization of the national governance system. This paper analyzes the value of big data in network public opinion management and proposes the path choice of network public opinion management in the era of big data in the big data era. Public opinion management talents, perfecting the network public opinion dialogue expression mechanism through government information disclosure, exerting the quantitative analysis and processing functions of network public opinion data, improving the rapid response mechanism of network public opinion, formulating unified standards to standardize data usage, and breaking through the limitations of big data network public opinion analysis, Strengthen network public opinion management big data technology support.
\end{abstract}

\section{Introduction}

Internet public opinion refers to the social political attitudes, beliefs and values that the public generates and holds on public issues and social managers in a certain social space, through the network around the occurrence, development and change of intermediary social events. Internet public opinion is the sum of the expressions of beliefs, attitudes, opinions and emotions expressed by more people about various phenomena and problems in society. Internet public opinion has formed rapidly and has a huge impact on society. With the rapid development of the Internet on a global scale, online media has been recognized as the "fourth media" after newspapers, radio and television. The Internet has become one of the main carriers reflecting social grievances.

In the big data environment, the network public opinion has undergone tremendous changes in terms of data volume, complexity and speed of production. As the core subject of network public opinion governance, the government faces severe challenges in governance capabilities. In this context, using the thinking and technology of big data, in-depth exploration of network panoramic data and public opinion laws, innovation of network public opinion supervision system, guiding mode and governance, and building a harmonious and rational network public space has become the modernization of the national governance system. Important content.

\section{Values of Big Data in Internet Public Opinion Management}

The value of big data in network public opinion management is mainly reflected in the following three aspects:

(1) Public opinion prediction. The traditional method of network public opinion monitoring has a lag. If the government fails to guide the network hotspots in time, it will spread rapidly in a short period of time, causing huge controversy and discussion among netizens, which can easily lead to public crisis events. Utilize big data technology to effectively analyze related network public opinion, establish a simulated network public opinion evolution process, maximize the prediction of network public opinion, and guide the network public opinion to develop in the right direction. 
(2) Comprehensive public opinion. Network public opinion monitoring in the era of big data not only focuses on text content, but also on pictures, voice, video, and complex social relationships. Pay attention to the source of data information, including traditional media, website news posts, overseas media online editions, BBS, online communities, forums, Weibo, search engines, WeChat public account and blogs. Through the comprehensive analysis of network public opinion, the value of network public opinion big data analysis is realized.

(3) Quantitative statistics. Network public opinion monitoring in the era of big data requires its own big data and converts big data information into standard data, which is quantifiable and quantifiable information. Simulate and analyze the standard data to predict the trend of future network public opinion development. The netizens who counted the grievances of the network published the number of comments and used quantitative indicators to identify them. The information is quantified and passed to the public, speaking with real numbers.

\section{New Features of Internet Public Opinion in Big Data Era}

In the face of vast data like the vast sea, whether it is the spread of network public opinion or management, new features have emerged:

(1) New features of online public opinion communication. Improve the ability to analyze and predict public opinion information, from the initial simple collection of data to research and development, speed up the transition rate from "static collection" to "dynamic tracking", from the initial reflection to the problem solving, and finally realize the network public opinion. Predictive. Compared with the linear communication of traditional media, the network public opinion realizes the true meaning of two-way interactive communication, focusing on small and large, attaching importance to micro-power, mining information from big data and judging trends.

(2) New features of network public opinion management. Big data brings technical advantages to online public opinion management. Through deep mining, deconstruct and reconstruct massive information, find out the correlation between data, provide data support for the spread of network public opinion, and improve the decision-making process. Based on cloud computing technology, the value of big data is more easily explored, which helps to improve the trend judgment ability and data analysis level, optimize public opinion early warning, response, judgment and decision-making, and improve the accuracy of public opinion management decision-making.

\section{New Challenges of Internet Public Opinion in Big Data Era}

The network public sentiment in the era of big data has undergone new changes and brought new challenges, mainly reflected in the following four aspects:

(1) The role of traditional media has dropped significantly. In the era of big data, data exists in non-structural and semi-structural forms, presenting diversity characteristics, and the corresponding information dissemination presents a trend of decentralization and fragmentation. Weibo, WeChat and mobile clients have become the first source of information for people to understand news. In particular, WeChat with a large number of active users has lowered the threshold of web browsing and expression, and has become a new engine of public opinion, promoting the equalization of online discourse rights. At the same time, the role of traditional media such as newspapers, magazines and television has dropped significantly. Under the social media discourse environment of the rise of online public opinion, the top-down public opinion authority of the mainstream media is being gradually dispelled.

(2) The uncontrollability of the emergency discourse system is enhanced. In the social media based on big data, the emergent discourse system presents some characteristics that are different from the traditional paradox: the anonymity of the subject of public opinion and the extensiveness of the channels of participation, the unboundedness of the space of communication and the real-time convergence of opinions. The spontaneity of the issue and the uncertainty of the development of public opinion, the diversity of values and the criticality of value orientation, the misconduct of opinion expression and the polarization of group behavior. Therefore, the 
uncontrollability of the discourse system of emergencies has enhanced the spread of bad information and rumors, which has jeopardized the network environment and damaged people's trust in the network.

(3) Insufficient protection of network public opinion data rights. The data footprint of the formation and development of online public opinion, while providing support for network public opinion, brings data rights and data privacy protection issues. The own, use and disposal rights of the collected network public opinion data, as well as the openness and openness of the public opinion data are not clear enough. In the process of collecting and using the public opinion, people are always exposed to the "third eye". Under surveillance, personal data privacy and rights may be violated. From the perspective of regional structure, urban and rural structure, age group and academic level, the imbalance of netizens' structure is still serious. It is difficult to fully reflect the public interest expression of the public.

(4) The different forms of online public opinion data have increased the difficulty of supervision. Internet public opinion data, mainly based on news websites, online forums, blogs, Weibo, WeChat, mobile clients, etc., through text, pictures, sounds, videos and web pages and other information carriers, acting on lyric objects, different information carriers, and combined form produces different propagation effects. Big data visualization technology provides a realistic basis for multimedia communication of network public opinion. The diversified features of network public opinion information are presented and released through multimedia modality. The diversification of network public opinion sources, participation in popularization, information structure and communication mechanism are complicated, and the difficulty of network public opinion supervision is increased.

\section{Path Selection of Internet Public Opinion Management in Big Data Era}

Internet public opinion is a concentrated reflection of social conditions and public opinion, and an effective tool to test the government's public service capabilities. In the context of advancing the modernization of the national governance system and governance capacity, we must make good use of the thinking and technical framework of big data, innovate the regulatory system and governance model of online public opinion, and combine the monitoring data of online public opinion with the actual public opinion survey to reshape The new relationship between the government and civil society in the context of the Internet, resolve the contradictions of social reality, and create a good cyberspace. The path proposed in this paper is as follows:

(1) Constructing a multi-subject collaborative governance mechanism for network public opinion. The multi-subject collaborative management of the Internet public opinion refers to the mutual promotion of the spontaneous formation of the government, network enterprises, netizens, non-governmental organizations and other entities under the premise of mutual trust, with the aim of seeking common interests as the motive to promote the optimal use of resources. , point and face combination, highly ordered, maximize the realization of the public interest self-organization collaborative governance structure. This method can effectively negotiate the relationship between the various governance bodies and promote the active participation of the subjects in complex network public opinion governance. At present, the multi-agent collaborative governance of online public opinion is mainly reflected in the synergy of resources in the decision-making of network public opinion governance and resource coordination in decision-making execution, and the policy synergy in governance effectiveness guarantee.

(2) Cultivate network public opinion management talents in the era of big data. The network public opinion in the era of big data presents multi-dimensional characteristics, also puts forward multiple quality and skill requirements for management talents, including social discourse expression, social psychological depiction, social relationship presentation and social appeal prediction. It is necessary to cultivate the era of big data. Internet public opinion management talents. Coordinate the strength of domestic institutions of higher learning, research institutes and government departments, systematically sort out the talent catalogues required for network public opinion management and set up specialized data science disciplines to cultivate both data mining 
and mathematical modeling, as well as high learning ability and analytical ability. And the level of knowledge, across the disciplines of statistics, sociology, computer science, communication and information science.

(3) Improve the expression mechanism of online public opinion dialogue through government information disclosure. Relying on big data technology, breaking government sector division, data monopoly and "information island", opening government data resources according to market rules and risk levels, promoting the convergence and sharing of government data resources, and improving government's network public opinion governance and public Service Level. Utilize big data technology to build a cross-media platform and cross-departmental regional connectivity mechanism, form a three-dimensional government information disclosure system, and promote the institutionalization and normalization of government information disclosure. Keep abreast of the sensation of online public opinion information, and provide timely, comprehensive and reliable information to the public to satisfy the public's right to know. Support and encourage citizens to make full use of the network to express their own rights and interests and form a benign and orderly public opinion expression network environment.

(4) The function of quantitative analysis and processing of network public opinion data. Public opinion quantification is the premise and basis for the realization of big data value. Establish a network public opinion index system such as public index, early warning index and evolution law index, and realize the transition from simple public opinion information collection to data processing and visualization, from one-dimensional crisis response to all-round information service. Comprehensively assess multi-dimensional data such as the nature of the event, the development trend, the communication platform, the scope of communication, the opinions of netizens, and the social influence. Integrate the network information sources, quickly and accurately classify the public opinion level, and enable the government to intervene in the network public opinion in advance, thus launching different early warning plans. To achieve accurate management and precise control of network public opinion.

(5) Improve the rapid response mechanism of online public opinion. In the practice of network public opinion guidance, according to the life cycle and evolution law of network public opinion, pay close attention to the public opinion trend on key nodes, timely release authoritative information and announce the disposal situation, and improve the rapid response mechanism. Often the more important the event, the more ambiguous the information, the greater the effect of the rumor. When at least one of importance and ambiguity tends to zero, the rumor will be self-defeating. Sometimes it is impossible to control the "importance" of an event, but it can control the "fuzziness" of the event. The best way to clarify the rumor is to make the information transparent. In the process of public opinion guidance, it is necessary to report the facts and report the reasons carefully. Expressing the facts is to release the truth of the matter in the first place, to satisfy the people's right to know, to demonstrate the authority of the government, and to ensure the role of leading public opinion.

(6) Develop uniform standards to regulate data usage. Different data standards lead to different analysis results, which will result in risk indicator bias when applied to network public opinion monitoring. Therefore, it is necessary to establish a unified data usage system and data definition between departments. The standardized data definition can realize the simple management of public opinion, the standardization of business processes, and the unification of research and judgment indicators, so that the main body of the network public opinion monitoring, clear its own positioning, coordinate the work of all parties, and facilitate the collection, analysis and processing of data. The big data management organization should actively promote the establishment of a unified data standard that is universally recognized by all walks of life, and actively improve and integrate with international standards to facilitate data exchange and integration between the network public opinion management department and other social organizations.

(7) Breaking through the limitations of public opinion analysis of big data networks. The premise of playing the value of data in the era of big data is the innovative means of acquiring, analyzing and applying data. At present, there are still many problems in using big data technology to control 
network public opinion, which has become a technical obstacle to network public opinion management in the era of big data. In order to break through the limitations of big data network public opinion management, first of all, strengthen the research and development of core technologies and products, form a technical system that integrates network public opinion monitoring, analysis, judgment, early warning and guidance, and build a mature and reliable network public opinion management product system. Second, develop and apply unified data standards to break data isolation and "information silos". Finally, improving data collation, analysis and forecasting capabilities requires the Internet platform to provide sufficiently data support, combined with the strength of research institutions and universities to jointly develop more advanced analytical tools and methods.

(8) Strengthen network public opinion management big data technology support. Big data has the advantages of comprehensiveness, dynamics and openness, as well as difficulties such as low value density and fast propagation speed. Therefore, it is necessary to speed up technical research and improve data analysis technology and capabilities. First, data monitoring technology. Achieve comprehensive capture of data such as media, forums, blogs, Weibo and WeChat, as well as automatic recognition of audio and video data. The second is data storage technology. Construct a mass storage big data platform to achieve efficient reading, writing and exchange of large-scale data. Third, data mining technology quickly identifies valuable data from massive data and mines the hidden laws behind the data. Fourth, data analysis techniques, including correlation analysis, cluster analysis and semantic analysis, automatically analyze the opinions and relevance of online speech, and reveal the development trend of public opinion. Fifth, data security technologies, including authentication, intrusion detection, and network protection, ensure data security.

\section{Acknowledgement}

This work is supported by 2018 annual natural science foundation in Liaoning province (20180550541): Research on Analysis Technology and System for Internet Public Opinion of Emergency in Big Data Environment.

\section{References}

[1] X. G. Li, "The judgment and guidance of network public opinion in the era of big data," Network Security Technology \& Application, vol. 16, no. 12, pp. 81-83, 2016.

[2] H. Y. Xiao, X. F. Zhu, "Systematic governance strategy of Internet public opinion under the national big data strategy," Journal of Tianshui College of Administration, vol. 18, no. 2, pp. 39-42, 2017.

[3] Y. Jin, H. Y. Xu, "On the System Construction of Government's Governance of Internet Public Opinion in the Era of Big Data Era," Journal of the Party School of Tianjin Committee of the CPC, vol. 20, no. 1, pp. 78-83, 2018.

[4] Z. Li, "Governance of Internet public opinion in big data environment," Youth Journalist, vol. 77, no. 2, pp. 76-77, 2017.

[5] Y. L. Li, G. Li, "Coping Strategy and Analysis of Online Public Opinion Emergencies in Big Data," Computer Knowledge and Technology, vol. 11, no. 15, pp. 14-15, 2015.

[6] J. Zhou, "Research on Internet public opinion under the background of big data," China Radio \& TV Academic Journal, vol. 30, no. 7, pp. 136-141, 2016.

[7] L. S. Xing, "Research on the change of Internet public opinion in the era of big data," Journal of Education and Media Studies, vol. 2, no. 4, pp. 42-44, 2017.

[8] X. X. Ning, "Big data thinking guides Internet public opinion analysis," Gansu Science and Technology, vol. 33, no. 10, pp. 13-14, 2015. 